\title{
UV Spectrophotometric Determination of Allopurinol and Benzbromarone in their Binary Mixture using Artificial Neural Networks and Genetic Algorithm- Artificial Neural Networks
}

\author{
Mohammad Wafaa I. Nassar, Khalid Abdel-Salam M. Attia, Ahmed Abdelhalim A. Mohamad, \\ Ragab Ahmad M. Said, Rady Fathy A. Gaber \\ Analytical Chemistry Department, Faculty of Pharmacy, Al-Azhar University, Nasr city, Cairo, Egypt
}

*Corresponding Author: Rady Fathy A. Gaber, Analytical Chemistry Department, Faculty of Pharmacy, Al-Azhar University, Nasr city, Cairo, Egypt.

\begin{abstract}
Chemometric assisted spectrophotometric models were suggested for the selective quantitative determination of allopurinol and benzbromarone without previous separation. Multivariate artificial neural networks and genetic algorithm artificial neural networks were developed. The proposed methods were validated and successfully applied for the determination of the drugs in their commercial preparation.
\end{abstract}

Keywords: Allopurinol, artificial neural networks, benzbromarone, genetic algorithm, multivariate calibration methods, pharmaceutical tablets.

\section{INTRODUCTION}

Allopurinol (ALP), figure (1), is (1, 5-Dihydro-4H-Pyrazolo [3, 4-d] pyrimidin-4-one) ${ }^{(1)}$ It is used in gout and hyperuricemia to inhibit the enzyme xanthine oxidase, thus preventing the oxidation of hypoxanthine to xanthine and xanthine to uric acid resulting in the reduction of urate and uric acid concentrations in plasma and urine. ${ }^{(2)}$

Benzbromarone (BENZ), figure (2), is (3, 5-dibromo-4-hydroxyphenyl)- (2-ethyl-3-benzofuranyl) methanone. ${ }^{(1)}$ It is a uricosuric drug that reduces plasma concentrations of uric acid by blocking renal tubular reabsorption. ${ }^{(2)}$

Combination of ALP and BENZ has the advantages of greater therapeutic effect than with either drug alone. This combination causes manifold reduction in uric acid concentrations in plasma and urine as compared to double dose of the individual drug when used alone. Also, this combination helps to decrease the dose of each active ingredient, and as a result, decreases the side effects of each of component if given separately in high doses. ${ }^{(3)}$

Reviewing the literature in hand, only four reports have been published for determination of the studied mixture, the first and second reports manipulated UV spectrophotometric methods. ${ }^{(3,4)}$ The third and fourth reports manipulated TLC-densitometric and RP-HPLC methods. ${ }^{(5,6)}$

The developed work aimed to develop and validate simple and sensitive chemometric assisted spectrophotometric methods for the simultaneous determination of ALP and BENZ in powdered forms, laboratory prepared mixtures and in pharmaceutical formulation namely, artificial neural networks and genetic algorithm artificial neural networks. The proposed methods have been optimized and validated as per the International Conference on Harmonization (ICH) guidelines ICH, and were found to comply with the acceptance criteria. ${ }^{(7)}$

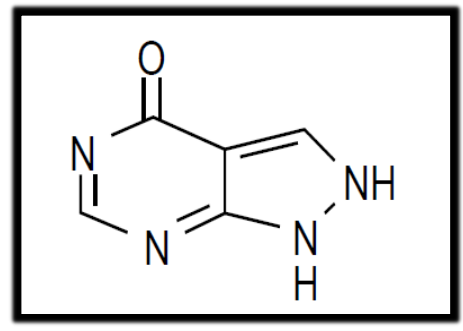

Figure3. Structural formula of $A L P$ 


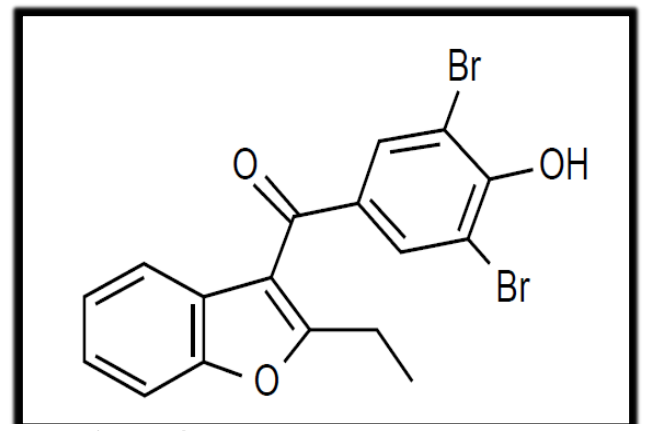

Figure4. Structural formula of BENZ

\section{EXPERIMENTAL}

\subsection{Materials}

\subsubsection{Pure Samples}

Pure ALP and BENZ standard samples (99.36 and $99.92 \%$ for ALP and BENZ, respectively), were kindly supplied by Global Napi Pharmaceuticals, 2nd Industrial Zone, 6th of October City- Egypt.

\subsubsection{Pharmaceutical Preparation}

Alloben $^{\circledR}$ tablets (100/25) (B.NO. B 10601) labeled to contain 100 mg ALP + 25 mg BENZ and were manufactured by Global Napi Pharmaceuticals, 2nd Industrial Zone, 6th of October City- Egypt, were purchased from a local market.

\subsubsection{Chemicals and Reagents}

Methanol, analytical grade (El-Nasr Company, Egypt).

\subsubsection{Apparatus}

- Shimadzu UV-Visible 1800 Spectrophotometer, (Tokyo, Japan), equipped with $10 \mathrm{~mm}$ matched quartz cells. Scans were carried out in the range from 200 to $400 \mathrm{~nm}$ at $0.1 \mathrm{~nm}$ intervals.

- Sonicator (Q sonica, LLC,53 Church Hill Road Newtown, CT.U.S.A).

\subsubsection{Standard Solutions}

Stock standard solutions for both ALP and BENZ containing $1000 \mu \mathrm{g} / \mathrm{mL}$ for each drug were prepared separately in methanol. Working standard solutions of both drugs $(100 \mu \mathrm{g} / \mathrm{mL})$ were obtained by dilution of the respective stock solutions with methanol.

\subsubsection{Software}

- UV-Probe personal spectroscopy software version 2.1. (Shimadzu).

- All chemometric methods were implemented in Matlab R2013b (8.2.0.701).

- All models were carried out by PLS toolbox software version 2.1.

\subsection{Procedures}

\subsubsection{Experimental Design}

Experimental design has been carried out using multilevel multifactor for construction of the calibration and validation sets ${ }^{(8)} .25$ samples were constructed based on five-levels, two factors. These 25 samples were prepared by transferring different volumes of ALP and BENZ from their standard solutions $(100 \mu \mathrm{g} / \mathrm{mL})$ into $10-\mathrm{mL}$ volumetric flasks and completed to volume with methanol. The choice of concentrations was based on the linearity range, $(2-16 \mu \mathrm{g} / \mathrm{mL})$ of ALPand $(1-16 \mu \mathrm{g} / \mathrm{mL})$ of $\mathrm{BENZ}$, and the ratio of the two drugs in the pharmaceutical preparation. The central level of the experimental design was $10 \mu \mathrm{g} / \mathrm{mL}$ of both ALP and BENZ. The absorption spectra of different ratios of the prepared mixtures were recorded over the wavelength range 200-400 nm with $1 \mathrm{~nm}$ interval. The region from 200-219 $\mathrm{nm}$ accounted for the rejection of this part from the spectra. 
ANN and GA-ANN models were constructed by transferring the recorded spectral data to Matlab ${ }^{\circledR}$ version R2013b (8.2.0.701), together with PLS-Toolbox 2.1. Software to apply the data analysis. 13 mixtures of this design were used as a calibration set and the other 12 mixtures were used as a validation set. The predictability of the proposed multivariate models was evaluated.

\subsubsection{Procedure for Pharmaceutical Preparation}

Ten Alloben ${ }^{\circledR}$ tablets were accurately weighed and finely powdered, then a quantity equivalent to 100 $\mathrm{mg}$ of ALP and $25 \mathrm{mg}$ of BENZ were extracted three times with $25 \mathrm{ml}$ of methanol by mixing well for 10 minutes by vigorous shaking then the prepared solution was sonicated for 20 minutes, finally the prepared solution has been filtered through Whatman filter paper No. 41 into 100 ml-volumetric flask. Filter paper was washed with methanol, adding washings to the volumetric flask and the volume was made up to the mark with methanol to obtain stock solution $(1000 / 250 \mu \mathrm{g} / \mathrm{mL})$. Working solution $(100 / 25 \mu \mathrm{g} / \mathrm{mL})$ was obtained by further dilution with methanol, and then analyzed using the general procedures of the proposed methods.

\subsubsection{Reported Method}

Dual wavelength, in which two wavelengths were selected for each drug in such a way that the difference in absorbance was zero for the second drug. At wavelengths 238.2 and $261.2 \mathrm{~nm}$ ALP had equal absorbance values; therefore, these two wavelengths have been used to determine BENZ; on a similar basis 253 and $274.4 \mathrm{~nm}$ were selected to determine ALP.

\section{RESULTS AND DISCUSSIONS}

In the present study, artificial neural networks and genetic algorithm artificial neural networks chemometric assisted spectrophotometric methods were applied for the determination of ALP and BENZ in their binary mixture in bulk powder and pharmaceutical preparation.

\subsection{Spectral Characteristics}

The zero-order absorption spectra of ALP and BENZ, as shown in figure (3), show severe overlap, which did not permit direct determination of them. So, the previously mentioned chemometric methods have been developed to resolve this overlapping and enable determination of ALP and BENZ without previous separation.

\subsection{Optimization of Experimental Conditions}

Initially constructing the calibration set for the binary mixture of the studied drugs was done to design the described models. The proposed methods were optimized with the aid of five-levels, two factors experimental design ${ }^{(8)}$ resulting in 25 sample mixtures. These 25 sample mixtures table (1) were split to two groups. The first group was 13 mixtures which were used to construct a calibration set. Another group was 12 mixtures were to compute the predictive benefits of the model. The choice of concentrations was based on the linearity range of each drug and the ratio of the two drugs in the pharmaceutical preparation. The selected wavelength range and the used spectral mode enhance the quality of the analysis. So, the recorded spectral data was pre-processed and the region 200-219 nm was rejected due to non-absorbance behavior, this results in 181 variables.

For the optimization of a neural network, a trial and error method has to be used to find the best neural network architecture. Selecting the optimum parameter values for constructing a network is not an easy task. In fact, the parameters are mutually related, so a compromise must usually be adopted. The error function RMSEP used as criterion for finalizing the learning process ${ }^{(9)}$.

The output layer is the concentration matrix of each component. The hidden layer consists of just a single layer which has been considered sufficient to solve similar or more complex problems. Moreover, more hidden layers may cause over fitting.

Among other ANN parameters, the hidden neurons number which is related to the converging performance of the output error function during the learning process. A grid search was done. The resulting error in prediction was the optimization criterion in this selection procedure. Another parameter that should be optimized carefully is the transfer function pair. Choosing of transfer function depends on the nature of data being analyzed. In this work, purelin-purelin transfer function was implemented due to linear correlation between absorbance and concentration. The learning rate controls the degree at which connection weights are modified during the learning phase. For 
UV Spectrophotometric Determination of Allopurinol and Benzbromarone in their Binary Mixture using Artificial Neural Networks and Genetic Algorithm- Artificial Neural Networks

optimization of these parameters, many experiments have been done to improve the model performance. The values of the optimized ANN parameters for ALP and BENZ were shown in table (2)

However, with the aim of increasing the quality of the calibration, wavelengths selection was performed, in such a way that uninformative variables were eliminated. This variables selection was carried out using the GA procedure. The GA was run on 181 variables for ALP and BENZ using a PLS with the maximum number of latent variables determined by cross-validation on the model containing all the variables. The adjusted GA parameters were shown in table (2).

GA reduced absorbance matrix to about 38.674 and $29.281 \%$ of the original matrix (70 and 53 variables for ALP and BENZ, respectively). The selected variables were used as input layer to ANN model. A large number of nodes in the input layer of the network (wavelengths) increase the CPU time for ANN modeling. GA allowed the use of less number of neurons (shorter training time) than those used in the network built with the raw data.

Recoveries, mean, SD, RMSEC and RMSEP and other validation parameters ALP and BENZ in both models were shown in table (3)

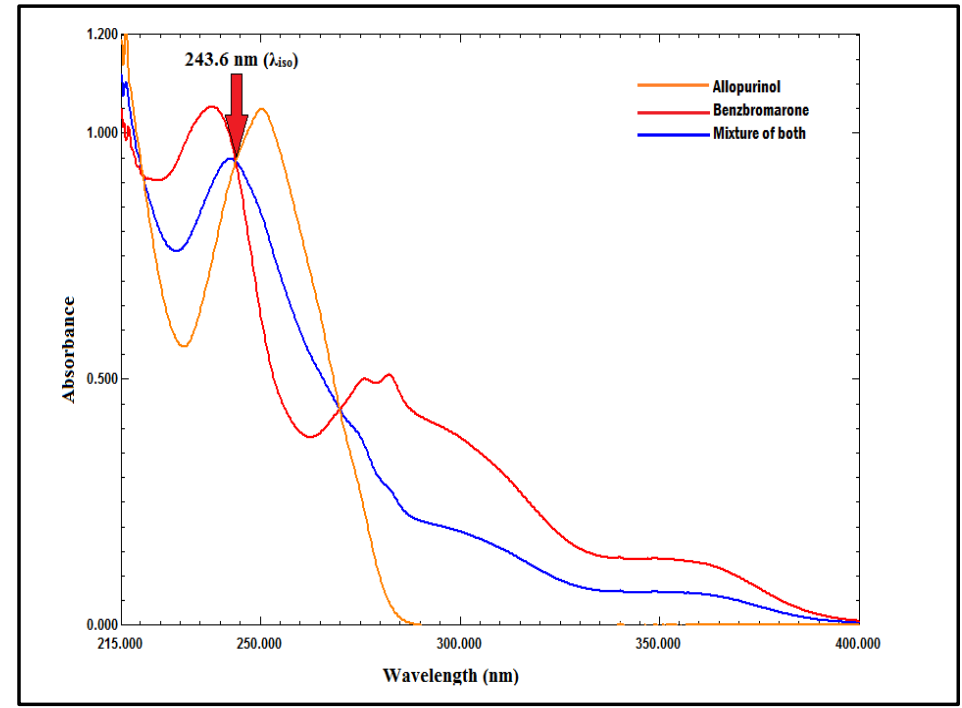

Figure3. Absorption spectra of $A L P(16 \mu \mathrm{g} / \mathrm{mL}), B E N Z(16 \mu \mathrm{g} / \mathrm{mL})$ and their mixture $(8+8 \mu \mathrm{g} / \mathrm{mL})$ of each .

\subsection{Pharmaceutical Application}

The proposed chemometric assisted spectrophotometric methods were applied for the determination of ALP and BENZ in Alloben ${ }^{\circledR}$ tablets. Satisfactory results were obtained in good agreement with the label claimed, indicating no interference from excipients and additives. The obtained results were statistically compared to those obtained by the reported method ${ }^{(3)}$. No significant differences were found by applying student's $t$-test and $F$ value at $95 \%$ confidence level ${ }^{(10)}$, indicating good accuracy and precision of the proposed method for the analysis of the studied drugs in their pharmaceutical dosage form, as shown in table (4).

Table1. Experimental design of concentrations of ALP and BENZ mixtures used in the chemometric assisted spectrophotometric methods

\begin{tabular}{|c|c|c|}
\hline No. of Mix & ALP $(\mu \mathrm{g} / \mathrm{mL})$ & BENZ $(\mu \mathrm{g} / \mathrm{mL})$ \\
\hline 1 & 10 & 10 \\
\hline 2 & 10 & 8 \\
\hline 3 & 8 & 12 \\
\hline 4 & 8 & 9 \\
\hline 5 & 12 & 12 \\
\hline 6 & 9 & 10 \\
\hline 7 & 12 & 9 \\
\hline 8 & 10 & 11 \\
\hline 9 & 9 & 12 \\
\hline 10 & 9 & 9 \\
\hline 11 & 11 & \\
\hline
\end{tabular}


UV Spectrophotometric Determination of Allopurinol and Benzbromarone in their Binary Mixture using Artificial Neural Networks and Genetic Algorithm- Artificial Neural Networks

\begin{tabular}{|c|c|c|}
\hline 12 & 12 & 11 \\
\hline 13 & 11 & 10 \\
\hline 14 & 10 & 12 \\
\hline 15 & 12 & 12 \\
\hline 16 & 12 & 8 \\
\hline 17 & 8 & 11 \\
\hline 18 & 11 & 10 \\
\hline 19 & 8 & 11 \\
\hline 20 & 10 & 11 \\
\hline 21 & 11 & 9 \\
\hline 23 & 11 & 8 \\
\hline 24 & 9 & 9 \\
\hline 25 & 8 & 10 \\
\hline
\end{tabular}

The shaded rows represent the calibration set.

Table2. Optimized parameters of ANNs and GA-ANNs for ALP and BENZ

\begin{tabular}{|c|c|c|c|c|}
\hline Methods & \multicolumn{2}{|c|}{ ANNs } & \multicolumn{2}{c|}{ GA-ANNs } \\
\hline Drug & ALP & BENZ & ALP & BENZ \\
\hline Architecture & $181-7-1$ & $181-6-1$ & $70-5-1$ & $53-4-1$ \\
\hline Hidden neurons number & 7 & 6 & 5 & 4 \\
\hline Transfer functions & \multicolumn{5}{|c|}{ Purelin - Purelin } \\
\hline Learning rate & 0.1 & 0.1 & 10 & 10 \\
\hline Training function & \multicolumn{4}{|c|}{ TRAINLM } \\
\hline
\end{tabular}

Table3. \% Recoveries, mean, SD, RMSEC and RMSEP for ALP and BENZ in the calibration and the validation samples by ANNs and GA-ANNs models

\begin{tabular}{|c|c|c|c|c|c|c|c|c|}
\hline \multirow{2}{*}{ Mixtures } & \multicolumn{4}{|c|}{ Calibration set } & \multicolumn{4}{c|}{ Validation set } \\
\cline { 2 - 10 } & \multicolumn{2}{|c|}{ ALP } & \multicolumn{2}{c|}{ BENZ } & \multicolumn{2}{c|}{ ALP } & \multicolumn{2}{c|}{ BENZ } \\
\cline { 2 - 9 } & ANN & GA-ANN & ANN & GA-ANN & ANN & GA-ANN & ANN & GA-ANN \\
\hline 1 & 100.00 & 98.74 & 99.99 & 100.07 & 102.17 & 100.23 & 100.25 & 101.69 \\
\hline 2 & 100.00 & 99.76 & 99.98 & 100.33 & 100.25 & 99.34 & 100.00 & 98.21 \\
\hline 3 & 100.00 & 99.37 & 100.00 & 98.93 & 100.56 & 98.03 & 104.42 & 97.02 \\
\hline 4 & 104.71 & 99.65 & 103.35 & 100.19 & 98.82 & 97.97 & 98.57 & 98.41 \\
\hline 5 & 100.00 & 97.96 & 99.97 & 100.28 & 100.67 & 99.06 & 100.18 & 97.98 \\
\hline 6 & 100.00 & 98.33 & 100.03 & 100.28 & 102.67 & 97.54 & 99.81 & 101.63 \\
\hline 7 & 100.00 & 99.81 & 100.00 & 100.15 & 96.03 & 98.93 & 100.25 & 99.36 \\
\hline 8 & 100.00 & 98.79 & 99.08 & 100.23 & 101.00 & 100.05 & 100.75 & 102.09 \\
\hline 9 & 100.25 & 101.17 & 96.79 & 99.82 & 99.82 & 100.68 & 104.03 & 100.95 \\
\hline 10 & 100.00 & 100.49 & 96.26 & 99.92 & 98.00 & 98.66 & 99.84 & 98.50 \\
\hline 11 & 100.21 & 100.44 & 100.02 & 100.20 & 101.09 & 99.38 & 101.64 & 101.43 \\
\hline 12 & 99.14 & 99.32 & 99.95 & 99.50 & 100.25 & 100.89 & 98.31 & 100.79 \\
\hline 13 & 100.00 & 100.14 & 99.98 & 99.83 & ------ & ------- & --------- & ------- \\
\hline Mean & 100.33 & 99.54 & 99.65 & 99.98 & 100.11 & 99.23 & 100.67 & 99.84 \\
\hline SD & 1.3429 & 0.919 & 1.700 & 0.396 & 1.809 & 1.083 & 1.877 & 1.773 \\
\hline RMSE & 0.165 & 0.104 & 0.180 & 0.036 & 0.182 & 0.134 & 0.194 & 0.179 \\
\hline
\end{tabular}

Table4. Determination of ALP and BENZ in Alloben ${ }^{\circledR}$ tablets by the proposed spectrophotometric and reported methods

\begin{tabular}{|c|c|c|c|c|c|c|}
\hline \multirow{2}{*}{ Parameters } & \multicolumn{2}{|c|}{ ANN } & \multicolumn{2}{c|}{ GA-ANN } & Reported & method $^{(3)}$ \\
\cline { 2 - 6 } & ALP & BENZ & ALP & BENZ & ALP & BENZ \\
\hline $\mathrm{n}^{*}$ & 5 & 5 & 5 & 5 & 5 & 5 \\
\hline Average (\%Recovery) & 99.70 & 101.59 & 101.17 & 101.57 & 99.99 & 100.68 \\
\hline \%RSD & 1.284 & 0.661 & 0.595 & 1.559 & 1.340 & 1.570 \\
\hline Student's $t$-test (2.306) & 0.349 & 1.188 & 1.794 & 0.892 & & \\
\hline F value (6.388) & 0.913 & 0.181 & 0.202 & 1.003 & & \\
\hline
\end{tabular}

*Number of samples

** The values in parenthesis are tabulated values of " $t$ " and " $F$ " at $(P=0.05)$

International Journal of Advanced Research in Chemical Science (IJARCS) 
UV Spectrophotometric Determination of Allopurinol and Benzbromarone in their Binary Mixture using Artificial Neural Networks and Genetic Algorithm- Artificial Neural Networks

\section{CONCLUSION}

The proposed multivariate calibration methods were simple, rapid, sensitive and precise and could be easily applied in quality-control laboratories for the simultaneous determination of ALP and BENZ in pure bulk powders. Moreover, these methods could be applied for dosage form analysis as well as in pure powder form without any preliminary separation step.

\section{ACKNOWLEDGEMENT}

I hope to thank my professors and my staff at analytical chemistry department, Al-Azhar University, Nasr city, Cairo, Egypt in helping me at everything.

\section{REFERENCES}

[1] British pharmacopoeia 2013. Vol. 2. London, UK: The stationery office; 2012.

[1] Sweetman S. Martindale: the complete drug reference. 36th ed. London: The Pharmaceutical Press; 2009.

[2] Ali NW, Abdelwahab NS, El-Fatatry HM, Osman WM. Spectrophotometric methods for simultaneous determination of two hypouricemic drugs in their combined dosage form. Pharm Anal Acta. 2013; 4(6): 18.

[3] Nassar MW, Attia KA, Abouserie AA, Said RA, Abdel-Kareem RF. Development and validation of UV spectrophotometric methods for simultaneous quantitative estimation of two hypouricemic drugs in their bulk powder and combined solid dosage form. Int J Pharm Pharm Res. 2017;11 (1):433-452.

[4] El-Fatatry HM, Osman WM.Development and validation of different chromatographic methods for determination of two hypouricemic drugs in their combined dosage form. Int $\mathbf{J}$ Adv Res Chem Sci. 2014;1(7):47-56.

[5] Ali NW, Abdelwahab NS, El-Fatatry HM, Osman WM.Development and validation of different chromatographic methods for determination of two hypouricemic drugs in their combined dosage form. $\mathrm{J}$ Anal Bioanal Tech. 2014; 5(5):1-8.

[6] International Conference on Harmonization, ICH Harmonised Tripartite Guideline. Validation of analytical procedure: text and methodology, Q2 (RI). Geneva: International Conference on Harmonization; 2005.

[7] Brereton RG. Multilevel multifactor designs for multivariate calibration. Analyst.1997; 122:1521-9.

[8] Yehia AM, Mohamed HM. Chemometrics resolution and quantification power evaluation: Application on pharmaceutical quaternary mixture of paracetamol, guaifenesin, phenylephrine and p-aminophenol. Spectrochim Acta A Mol Biomol Spectrosc. 2016; 152:491-500.

[9] Armitage P, Berry G. Statistical methods in medical research. 3rd ed. Oxford (UK): Blackwell; 1994.

Citation: M. Nassar et al., "UV Spectrophotometric Determination of Allopurinol and Benzbromarone in their Binary Mixture using Artificial Neural Networks and Genetic Algorithm-Artificial Neural Networks", International Journal of Advanced Research in Chemical Science (IJARCS), vol. 5, no. 8, pp. 24-29, 2018. http://dx.doi.org/10.20431/2349-0403.0508005

Copyright: () 2018 Authors. This is an open-access article distributed under the terms of the Creative Commons Attribution License, which permits unrestricted use, distribution, and reproduction in any medium, provided the original author and source are credited. 